\title{
Sleep and Pain: A Relationship that Begins in Early Life
}

A complex interaction of genetic and environmental factors modulates pain sensitivity. The early years' experiences are an important environmental factor that has been associated to predispose an individual to pain conditions. Prolonged postnatal brain maturation makes the early years a critical period for adequate development and suggests that insults in infancy may cause long-term alterations in pain sensitivity $(1,2)$. The vulnerability of the pain system during development indicates the need to investigate infant's experiences as a possible determinant of pain conditions.

Sleep quality and quantity can modulate pain sensitivity. Reduction in sleep time increases pain response and chronic pain conditions are able to alter sleep pattern $(3,4)$. This relationship is well documented in adults, but very little is known about how pain and sleep interacts in early life. Sleep is a predominant behavior during infancy and evidence indicates that sleep is essential for brain development (5-7). Whether sleep is crucial for the maturation of several physiological systems, including the pain system, the bidirectional relation between sleep and pain begins early in life.

To better elucidate the function of sleep in early years and the relation between sleep and pain we conducted an investigation in mice to evaluate the effect of neonatal sleep deprivation on nociceptive behavior, an important component of the pain system. We hypothesized that neonatal sleep loss could be a threat to the developing organism, leading to long-term changes in pain sensitivity. An animal model is a reliable tool to explore behavioral impairment induced by sleep deprivation under controlled experimental conditions.

Neonatal mice at postnatal day (PND) 12 were randomly assigned to the following groups: control (CTRL), sleep deprivation (SD) or maternal separation (MS). Sleep deprivation was conducted using the gentle handling method. The method of gentle handling involves touching the animals with the hand or a brush, or shaking the cage, when pups showed signs of sleep onset. Sleep was behaviorally detected by motor activity quiescence, eye closure and myoclonic twitching. The gentle handling method is effective in producing SD in neonatal rodents, being able to eliminate up to $91 \%$ of total sleep time (8). The manipulation of the pups occurred in the absence of the mother. To control for the possible effects of the absence of the mother, the MS group was used. In the MS group, the litters were removed and placed in separate cages, which were put on a heating pad set in an adjacent room. During the separation period, food and water was not available, pups were not manipulated and could sleep. The SD and MS were only conducted for a period of 6 hours (from 8 a.m. to 2 p.m.). The CTRL litters remained undisturbed with dams in the colony room. All pups in the same litter received the same treatment. Weaning occurred at PND 22, when all pups were group housed by litter and sex until adolescence (PND 35, $\mathrm{n}=7-9$ / group/sex) or adulthood (PND 90, $\mathrm{n}=6-8$ /group/sex). At each developmental time-point (PND 35 or PND 90), the mice were tested for nociceptive response using the hot plate test. In this test mice were placed singly on a hot plate apparatus (Ugo Basile, Italy) that consists of a temperature-controlled metal surface $\left(53 \pm 1^{\circ} \mathrm{C}\right)$ encased by a cylindrical Plexiglas chamber. A timer was started when all 4 paws were in contact with the surface. The latency to withdraw the paw (licking either a hind paw or forepaw) to avoid thermal stimulus was measured in seconds, at which point the mouse was immediately removed from the hot plate and the timer was stopped. The paw withdrawal threshold to avoid thermal stimulus is a widely used measure of nociceptive response in rodents. Sixty second cut-off latency was determined for non-responsive animals (9). The experimental protocol was in accordance with the Ethical Guidelines for Investigations of Experimental Pain in Conscious Animals (10) and it was approved by the University's Ethics Committee (\#1960/09). Data were evaluated using two-way analysis of variance (ANOVA), with group and sex as main factors. Post-hoc comparisons were performed using the Tukey's test whenever necessary. Results were expressed as the mean \pm S.D. The level of significance was set at $P<0.05$. All analyses were carried out using SPSS 20.

The long-term effects of neonatal manipulations on nociceptive response showed their main effect in the adolescent mice $(F(2,42)=3.97 ; P=0.02$; eta $=0.15$; power $=0.68$ ). Six hours of SD at PND 12 was able to increase nociceptive sensitivity at PND 35, with adoles- 
A

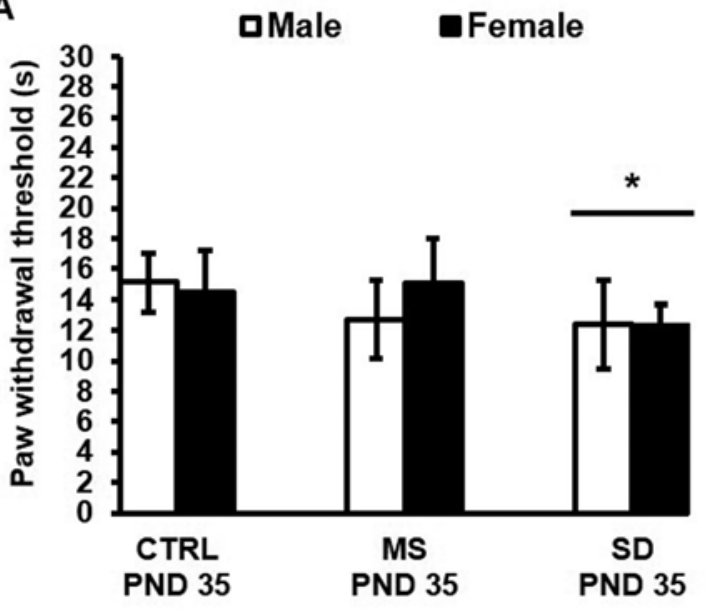

B

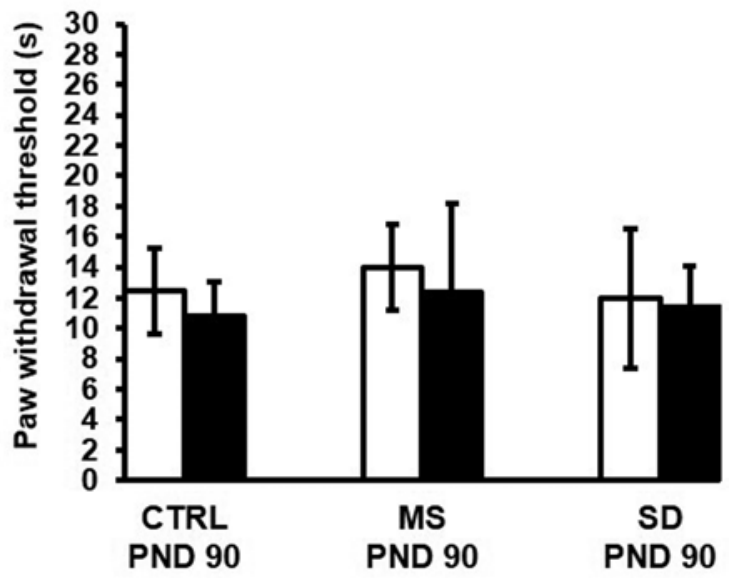

Fig. 1. Long-term effect of sleep deprivation on nociceptive response. A: Latency to withdraw the paw (licking either a hind paw or forepaw) to avoid thermal stimulus (hot plate) in mice at PND $35(A, N=7-9 /$ sex/group, total $N=48)$ and PND 90 (B, $N$ = 6-8/sex/group, total $N=46)$. CTRL: control; MS: maternal separation; SD: sleep deprivation; PND: postnatal day. Data are presented as mean \pm S.D. *vs. CTRL, $P<0.05$.

cent mice exhibiting a lower paw withdrawal threshold compared with CTRL mice (12.3 vs. $14.8 \mathrm{sec}, P=0.01$; Fig. 1A). Sleep loss in a critical period for pain system development reduced the nociceptive threshold by $17 \%$. There was no significant main effect or interactions on nociceptive response in adult mice. The paw withdrawal threshold at PND 90 was statistically similar between groups (Fig. 1B).

Nociception is an essential component of the pain system and dysfunctions of the nociceptive response, such as hypersensitivity, could be associated with increased risk for chronic pain conditions. In the present study, sleep deprived pups on PND 12 showed hypersensitivity during adolescence revealed by the lower paw withdrawal threshold on hot plate test, even after 3 weeks of normal sleep conditions. The increased sensitivity could reflect a brain that is more responsive and vulnerable to noxious stimuli as a result of neonatal sleep loss. However, this change in nociceptive response was not statistically different from the MS group and it was not present in adult mice. It is possible that our results reflect a short-term effect of the accumulation of manipulations (summation of sleep deprivation associated to maternal separation), not only sleep loss per se. Despite this limitation, our data add evidence to a scarce literature related to sleep and pain interactions in early-life.

We believe that our data will encourage more animal and clinical studies to investigate the bidirectional relation between sleep and pain at different develop- mental time-points. Given the importance of sleep for health and well being, perhaps improved children's sleep could be the key to improve pain condition in pediatric populations.

\section{Acknowledgement}

Part of these data were presented in abstract format at the FENS conference, July 2012, and it was awarded with FENS/IBRO Travel Grant. Authors' contributions: study design (PA, MLA), data acquisition and analysis (PA), drafting manuscript and critical revision (PA, ST, MLA). All authors give final approval of the version. This study was supported by Research Fund Incentive Association (AFIP) and São Paulo Research Foundation (FAPESP \#2010/50130-0 to PA). MLA and ST are recipients of fellowships from $\mathrm{CNPq}$. The authors have no conflicts of interest.

Paula Araujo, PhD

Departamento de Psicobiologia

Universidade Federal de São Paulo

Rua Napoleão de Barros, 925

Vila Clementino - SP- 04024-002

Brazil

Sergio Tufik, MD, PhD

Departamento de Psicobiologia Universidade Federal de São Paulo

Rua Napoleão de Barros, 925

Vila Clementino - SP- 04024-002

Brazil 


\section{Monica Levy Andersen \\ Departamento de Psicobiologia - \\ Universidade Federal de São Paulo \\ Rua Napoleão de Barros, 925, \\ Vila Clementino - SP- 04024-002 \\ Brazil \\ E-mail: ml.andersen12@gmail.com}

\section{References}

1. Hohmeister J, Kroll A, Wollgarten-Hadamek I, Zohsel K, Demirakça S, Flor H, Hermann C. Cerebral processing of pain in school-aged children with neonatal nociceptive input: an exploratory fMRI study. Pain 2010; 150:257-267.

2. Schwaller F, Fitzgerald $M$. The consequences of pain in early life: Injury-induced plasticity in developing pain pathways. Eur ] Neurosci 2014; 39:344-352.

3. Smith MT, Haythornthwaite JA. How do sleep disturbance and chronic pain inter-relate? Insights from the longitudinal and cognitive-behavioral clinical trials literature. Sleep
Med Rev 2004; 8:119-132.

4. Edwards RR, Almeida DM, Klick B, Haythornthwaite JA, Smith MT. Duration of sleep contributes to next-day pain report in the general population. Pain 2008; 137:202-207.

5. Roffwarg HP, Muzio JN, Dement WC. Ontogenetic development of the human sleep-dream cycle. Science 1966; 152:604-619.

6. Graven S. Sleep and brain development. Clin Perinatol 2006; 33:693-706.

7. Sokoloff G, Uitermarkt BD, Blumberg MS. REM sleep twitches rouse nascent cerebellar circuits: Implications for sensorimotor development. Dev Neurobiol 2014, in press.

8. Hairston IS, Ruby NF, Brooke S, Peyron C, Denning DP, Heller HC, Sapolsky RM. Sleep deprivation elevates plasma corticosterone levels in neonatal rats. Neurosci Lett 2001; 315:29-32.

9. Sternberg WF, Scorr L, Smith LD, Ridgway CG, Stout M. Long-term effects of neonatal surgery on adulthood pain behavior. Pain 2005; 113:347-353.

10. Zimmerman M. Committee for Research and Ethical Issues of the IASP. Ethical guidelines for investigations of experimental pain in conscious animals. Pain 1983;16:109110. 